\title{
Broadcast Gossip Based Distributed Hypothesis Testing in Wireless Sensor Networks
}

\author{
The-Dung Pham, Hung Quoc Ngo, Viet-Duc Le, Sungyoung Lee, Young-Koo Lee \\ Department of Computer Engineering \\ Kyung Hee University \\ Yongin, Republic of Korea \\ \{ptdung,nqhung,levietduc,sylee,yklee\}@oslab.khu.ac.kr
}

\begin{abstract}
We consider the scenario that $\mathrm{N}$ sensors collaborate to observe a single event. The sensors are distributed and can only exchange messages through a network to reach a consensus about the observed event. In this paper, we propose a very robust and simple method using broadcast gossip algorithm to solve the distributed hypothesis testing problem. The simulation result shows that our method has good performance and is very energy efficient comparing to existing methods.
\end{abstract}

\section{INTRODUCTION}

Wireless sensor networks (WSNs) provide an inexpensive, convenient and powerful way for data gathering and environment monitoring. The dense deployment, resource constraints, and unattended nature of WSNs make the issue of energy efficiency a primary design goal in this field [1],[2].

We focus on the scenario in which there are $\mathrm{N}$ distributed sensors affected by noise observing a single event. The task is to reach a consensus in the network about the event after exchanging messages. This problem is known as distributed hypothesis testing (DHT). Each node makes a conditionally independent observation of the event and needs to know the most probable event based on all observations. For the purpose of energy saving, each sensor is limited to only communicate with its neighbors (sensors in its transmission radius). To deal with DHT problem, some novel approaches have been proposed recently. Alayani et al [3] used belief propagation (BP) [9] as a message passing strategy to solve the DHT problem for a pre-specified network connectivity. This method has several limitations. As mentioned in their paper, the algorithm may fail to converge or converge to an inaccurate estimate due to the unknown of asymptotic features in general network topology. In addition, in WSNs, network topology changes frequently because of obstacles, node failures, etc.; meanwhile, there exists no result on the convergence of belief propagation on networks with changing topologies. This approach therefore is not properly suitable for WSNs. Saligrama et al [11] introduced some modified belief propagation algorithms to limit each of BP drawbacks. Nevertheless, it still lacks a simple and unified solution to overcome those all drawbacks.

Recently, R. Olfati-Saber et al [4] proposed a belief consensus algorithm for solving the DHT problem. Belief consensus, based on theoretical results of consensus algorithms [5], is robust to link failures and converged under topology changes. However, the system of [5] is able to reach a consensus if and only if the common delay is smaller than a given, topology-dependent, value. Moreover, the assumption of homogeneous delays and symmetric links of consensus protocol is not appropriate for modeling the propagation delay in a typical WSN, where the delays are proportional to the distance between the nodes and communication channels are typically asymmetric.

In this paper, we propose a scheme using broadcast gossip algorithm [8] to solve the DHT problem. Broadcast gossip algorithm is a distributed, robust and simple algorithm that always ensures the accuracy and convergence with probability one for DHT problem. Further more, it has been proved to be well suited in WSNs in context of performance and energy efficiency.

The remainder of this paper is organized as follows. The average consensus-based DHT problem is formulated in Section II. In section III, we first briefly present the broadcast gossip algorithm [8], and then describe our proposed scheme combining the gossip algorithm and a maximum a posteriori (MAP) estimator to solve the DHT problem. Experimental results are presented in section IV. Section V concludes the paper.

\section{PROBLEM FORMULATION}

Consider a connected undirected graph, which represents the communication structure among sensors, $G=(V, E)$ with vertex set $V=\{1,2, \ldots, N\}$ and edge set $E \subset V \times V$. For each node $i \in V$, let $N(i)=\{j \mid(i, j) \in E\}$ be the set of neighbors of $i$, that is, the set of nodes within its radio range.

We focus on a Bayesian hypothesis testing problem, which involves a set $H=\left\{H_{1}, H_{2}, \ldots, H_{M}\right\}$ of hypotheses, along with a known prior probability distribution $\pi_{0}$ on $H$. We are interested in estimating a true hypothesis based on a collection of observations $Y=\left(Y_{1}, Y_{2}, \ldots, Y_{N}\right)$ where each entry represents measurements taken by a distinct sensor. Assuming that observations are conditionally independent given the true hypothesis. That is, the conditional distribution of the observation vector $\left(Y_{1}, Y_{2}, \ldots, Y_{N}\right)$ factors and it turns out that 
the posterior probability $\pi\left(H_{m}\right)$ of hypothesis $H_{m}$ based on these measurements satisfies

$$
\begin{gathered}
\pi\left(H_{m} \mid Y\right)=\alpha \pi_{0}\left(H_{m}\right) f\left(Y \mid H_{m}\right), \\
\pi\left(H_{m} \mid Y\right)=\alpha \pi_{0}\left(H_{m}\right) \prod_{i=1}^{N} f_{i}\left(Y_{i} \mid H_{m}\right), \\
m=1,2, \ldots, M .
\end{gathered}
$$

We need to compute a MAP estimate, which is the hypothesis with the largest posterior probability. In particular, hypothesis $H_{m^{*}}$ is a MAP estimate if

$$
\pi_{0}\left(H_{m^{*}}\right) \prod_{i=1}^{N} f_{i}\left(Y_{i} \mid H_{m^{*}}\right)=\max _{m}\left(\pi_{0}\left(H_{m}\right) \prod_{i=1}^{N} f_{i}\left(Y_{i} \mid H_{m}\right)\right) .
$$

In seminal work of Perl [10], the conditional probability $f_{i}\left(Y_{i} \mid H_{m}\right)$ is called the belief of node $i$ in the network. Our objective is to calculate the product of beliefs using distributed algorithm. Taking the logarithm of both sides of (1) leads to

$$
\begin{aligned}
\log \left(\pi\left(H_{m} \mid Y\right)\right) & \propto \log \left(\pi_{0}\left(H_{m}\right)\right)+\sum_{i=1}^{N} \log \left(f_{i}\left(Y_{i} \mid H_{m}\right)\right) \\
& \propto \log \left(\pi_{0}\left(H_{m}\right)\right)+N^{*} \underset{i \in V}{\operatorname{Ave}\left(\log \left(f_{i}\left(Y_{i} \mid H_{m}\right)\right)\right),}
\end{aligned}
$$

where Ave(.) denotes the average function.

Then the MAP estimate can be rewritten in the following form:

$$
\begin{aligned}
m^{*} & =\arg \max _{m} \log \left(\pi\left(H_{m} \mid Y\right)\right) \\
& =\arg \max _{m}\left[\log \left(\pi_{0}\left(H_{m}\right)\right)+N^{*} \underset{i \in V}{\left.A v e\left(\log \left(f_{i}\left(Y_{i} \mid H_{m}\right)\right)\right)\right]}\right.
\end{aligned}
$$

Thanks to (4), we can utilize a distributed averaging algorithm to estimate the average logarithm of every node belief and that will be used in the MAP estimation. We present our approach in the following section.

\section{DistRIBUtED HyPOTHESIS TESTING USING BROADCAST GossIP}

In this section, we first introduce the distributed averaging problem. Then we talk over broadcast gossip algorithm and how to apply it to the DHT problem.

\section{A. Distributed Average Consensus}

Let $x_{i}(0)$ be a real-valued number (or a vector) assigned to node $i$ at time $t=0$ as node state, representing an observation of some type. The distributed average consensus problem is to compute iteratively the average $(1 / N) \sum_{i=1}^{N} x_{i}(0)$ at all nodes, using only local state and communication with its neighbor nodes.
Much recent research has focused on various distributed averaging algorithms. Distributed averaging, also known as the distributed consensus problem, has been studied in context of consensus problem [5], Metropolis weights [10], gossip algorithms [6], [7], [8].

\section{B. Broadcast Gossip Algorithm}

Gossip is a well-known technique for distributed computing in an arbitrarily connected network of nodes. Gossip algorithm for distributed averaging problem has received much attention [6], [7], [8]. In context of this paper, we used asynchronous broadcast gossip algorithm [8] to solve the distributed averaging problem. This algorithm is proved to have better performance than others of [6], [7] when applying to WSNs.

Suppose at time step $t$-th, node $i \in\{1,2, \ldots, n\}$ clock ticks. Node $i$ is activated and the following events occur:

1. Node $i$ broadcasts its current state value, $x_{i}(t)$ over the network.

2. The broadcast value is successfully received by all neighbors $\mathrm{k} \in N(i)$.

3. Each neighbor $k \in N(i)$ uses the broadcast value $x_{i}(t)$ to update its own state value according to:

$$
x_{k}(t+1)=\gamma x_{k}(t)+(1-\gamma) x_{i}(t), \forall k \in N(i),
$$

where $\gamma \in(0,1)$ is the mixing parameter of the algorithm.

4. The remaining nodes in the network, including $i$, update their state value as

$$
x_{k}(t+1)=x_{k}(t), \forall k \notin N(i) .
$$

\section{Broadcast Gossip based DHT (BGDHT) algorithm}

We propose a new method to solve the DHT problem. First, broadcast gossip is used to calculate the average of logarithm of every sensor belief. Then a MAP estimator combines that result with the prior probability to determine the most probable hypothesis or event. This whole process is carried out by the BGDHT algorithm presented below.

Suppose that each node $i$ has measurement $Y_{i}$ of the observed event.

1. Each node computes a vector of logarithm of conditional probability

$$
x_{i}(0)=\log f_{i}\left(Y_{i} \mid H_{m}\right), m=1, \ldots, M .
$$

2. Apply broadcast gossip algorithm to achieve average vector $\bar{x}$.

3. Each node uses a MAP estimator to find out the most possible event

$$
m^{*}=\underset{m}{\arg \max }\left(\log \left(\pi_{0}\left(H_{m}\right)\right)+N \times \bar{x}[m]\right),
$$

where $\bar{x}[m]$ is the $m$-th element of vector $\bar{x}$. 


\section{The problem of network size}

The MAP estimate in (4) requires prior knowledge of the number of sensors $\mathrm{N}$. It will be simple if $\mathrm{N}$ is known at deploying phase of the WSN; otherwise we have to estimate it. We extend the broadcast gossip protocol above to let the WSN reach a consensus on the number of nodes $\mathrm{N}$, as follows. Assume it can be ensured that only one node has set $x_{i}(0)=1$ (e.g., the sink node), while all other nodes have set $x_{i}(0)=0$ at initialization. By using gossip algorithm, each node obtains the average value $\bar{x}=1 / n$, which is the reciprocal of the number of nodes.

Especially, in the case of equally prior probabilities $\pi_{0}\left(H_{i}\right)=\pi_{0}\left(H_{j}\right), \forall i, j \in\{1, \ldots, M\}$, i.e., all hypotheses are equally likely to happen, then we do not need to estimate the number of nodes because the MAP estimated in (4) becomes the maximum likelihood estimate (MLE):

$$
m^{*}=\underset{m}{\arg \max }\left[\underset{i \in V}{A v e}\left(\log \left(f_{i}\left(Y_{i} \mid H_{m}\right)\right)\right)\right] .
$$

Therefore (8) is reduced to simple form

$$
m^{*}=\underset{m}{\arg \max }(\bar{x}[m]) .
$$

\section{EXPERIMENTAL RESULTS}

To evaluate the BGDHT algorithm, we consider the problem of grid-based distributed target localization. In this problem, target is positioned at an unknown position in the sensor field and emits an isotropic signal. We would like to estimate the source location using received signal energy measurements taken at each sensor.

We assume that noises at local sensors are i.i.d. samples of a zero-mean Gaussian noise process $n_{i} \sim N\left(0, \sigma^{2}\right)$.

The received signal strength at sensor $i$ is modeled as $s_{i}=a_{i}+n_{i}, a_{i}$ is the signal amplitude and is determined by

$$
a_{i}=\sqrt{\frac{P_{0}}{1+\beta d_{i}^{2}}},
$$

where $P_{0}$ is the signal power emitted by the target at distance zeros, $\beta$ is an adjustable constant (larger $\beta$ implies faster signal power decay), and $d_{i}$ is the distance between the target and local sensor $i$ :

$$
d_{i}=\sqrt{\left(x_{i}-x_{t}\right)^{2}+\left(y_{i}-y_{t}\right)^{2}} .
$$

In our simulation, we chose the parameter values $\sigma=1, P_{0}=1000, \beta=1$.

As shown in figure 1, $\mathrm{N}$ sensors are uniformly deployed in a region of interest (ROI) which is a square with area $a^{2}$. The sensor field is divided into square-shaped grid having $N_{x} \times N_{y}$ cells. Each square block $H\left(h_{x}, h_{y}\right)$ is considered as a hypothesis for target location.

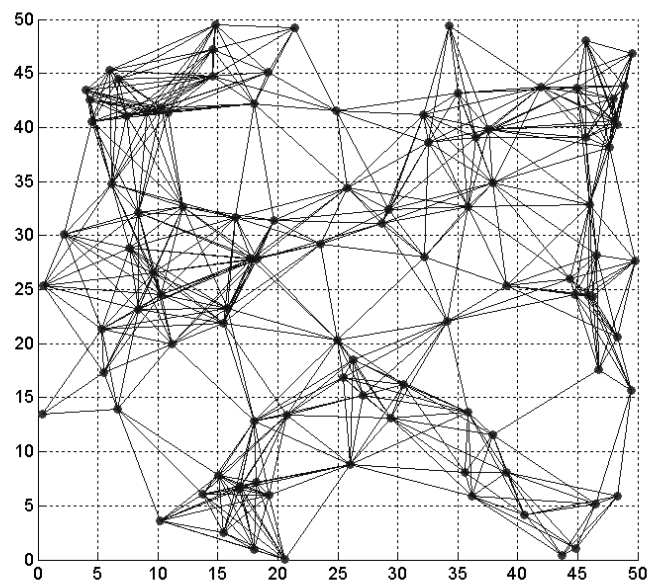

Figure 1. Uniform sensor deployment with $\mathrm{N}=100$ nodes.

Based on a set of $N_{t}=10000$ observations (training data), each sensor learns the statistical model of target location. The distribution of received signal strength given the target location is modeled by a Gaussian distribution

$$
s_{i} \sim N\left(\mu_{i}\left(h_{x}, h_{y}\right), \sigma_{i}^{2}\left(h_{x}, h_{y}\right)\right) .
$$

An example of these distributions is given in figure 2 .

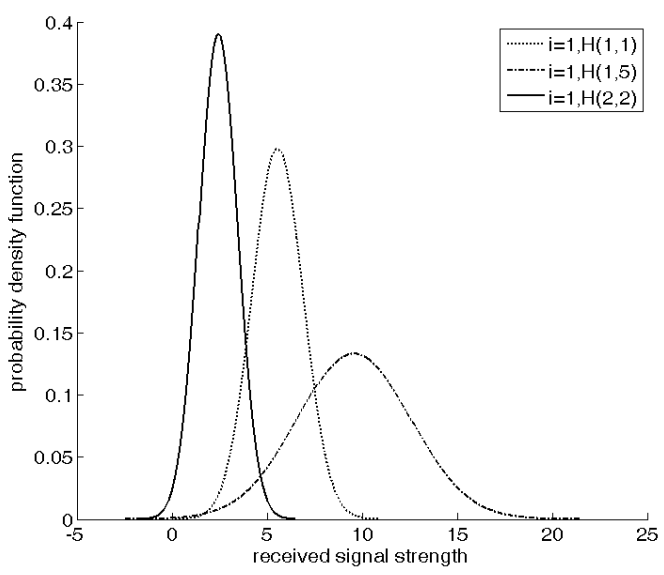

Figure 2. Gaussian distribution of received signal strength given target location.

In localization stage, after taking measurements, each node needs to calculate its beliefs. The probability that $s_{i}$ lies in an interval $\delta \ll 1$ can be approximated as

$$
p\left(s_{i} \mid H\left(h_{x}, h_{y}\right)\right) \approx \delta \frac{1}{\sqrt{2 \pi \sigma_{i}^{2}\left(h_{x}, h_{y}\right)}} \exp \left(\frac{\left(s_{i}-\mu_{i}\left(h_{x}, h_{y}\right)\right)^{2}}{\sigma_{i}^{2}\left(h_{x}, h_{y}\right)}\right) .
$$

Since $\delta$ is a positive constant, it will be eventually absorbed in the normalization constant of the posterior 
probability and therefore it can be dropped. Hence, we apply the following approximation for the beliefs calculated locally by every sensor according to its statistical model learned from training data:

$$
p\left(s_{i} \mid H\left(h_{x}, h_{y}\right)\right) \approx \frac{1}{\sqrt{2 \pi \sigma_{i}^{2}\left(h_{x}, h_{y}\right)}} \exp \left(\frac{\left(s_{i}-\mu_{i}\left(h_{x}, h_{y}\right)\right)^{2}}{\sigma_{i}^{2}\left(h_{x}, h_{y}\right)}\right) .
$$

In general, each sensor constructs a set of $N_{x} \times N_{y}$ beliefs which are input to our BGDHT algorithm. After some iterations, the sensors asymptotically reach a common set of beliefs that allow to choose the most likely hypothesis $H\left(h_{x}^{*}, h_{y}^{*}\right)$ corresponding to the target location.

Figure 3 depicts the convergence rate to consensus of our BGDHT, belief consensus, and Metropolis algorithm. It is obtained by averaging the total number of rounds incurred over 20 executions on a network of 100 nodes. As we can see, the BGDHT outperforms the others in terms of communication cost. The BGDHT requires fewer rounds of communications to reach a consensus than the others.

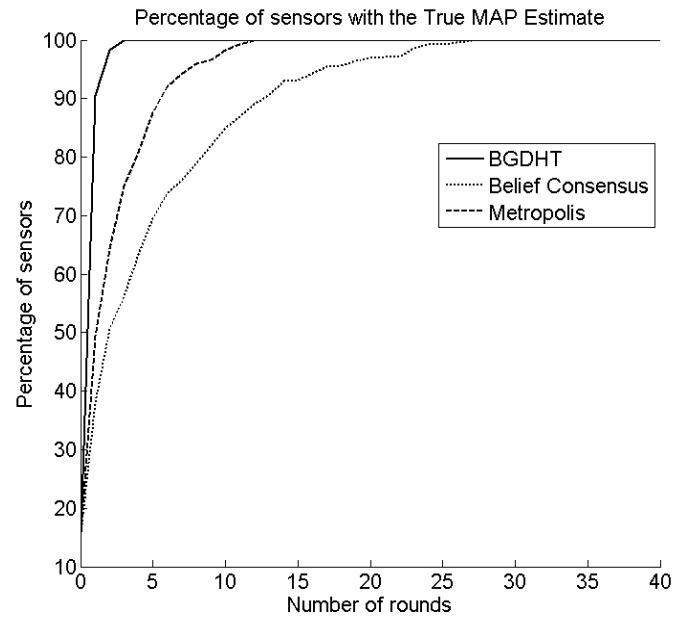

Figure 3. Rate of convergence to consensus.

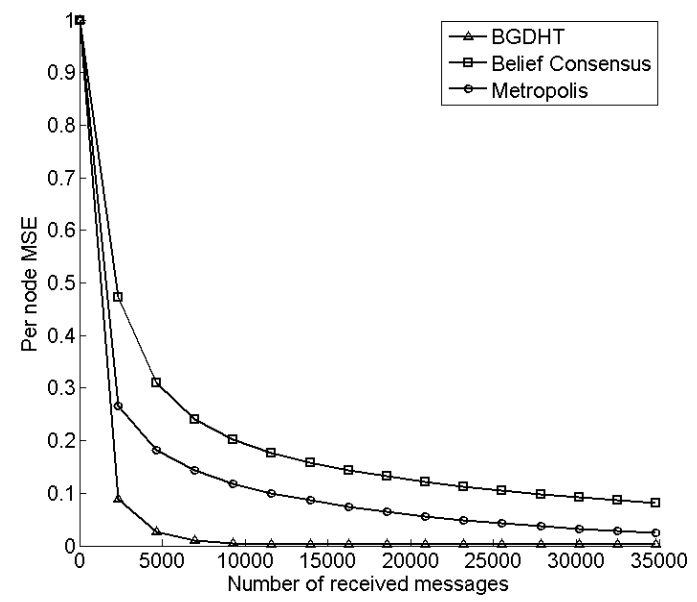

Figure 4. Number of transmission required to achieve a given MSE.
Next, we examine the mean square error (MSE) performance of the BGDHT algorithm through iterations and compare its performance to those of belief consensus and Metropolis algorithm. Figure 4 depicts the MSE versus the number of radio transmissions. Of note is that the simulation results are normalized by the largest corresponding output. The GHDHT also has better MSE performance than the others.

\section{CONCLUSION}

In this paper, we propose a new approach using broadcast gossip mechanism for distributed hypothesis testing problem in WSNs. Our approach does not suffer from limitations of previous methods. It performs well in terms of convergence speed and energy consumption. A detailed example is provided for distributed source localization using a network of sensors with self-constructed statistical models.

\section{ACKNOWLEDGMENT}

This research was supported by the MKE (Ministry of Knowledge Economy), Korea, under the ITRC (Information Technology Research Center) support program supervised by the IITA( Institute of Information Technology Advancement)" (IITA-2009-(C1090-0902-0002)) and Was supported by the IT R\&D program of MKE/KEIT, [10032105, Development of Realistic Multiverse Game Engine Technology]. This work also was supported by the Brain Korea 21 projects and Korea Science \& Engineering Foundation (KOSEF) grant funded by the Korea government(MOST) (No. 2008-1342).

\section{REFERENCES}

[1] Chee-Yee Chong and Srikanta P. Kumar, "Sensor networks: evolution, opportunities, and challenges". In Proceedings of the IEEE, Volume 91 No 8, pages 1247.1256, August 2003.

[2] Deborah Estrin, Ramesh Govindan, John Heidemann, and Satish Kumar, "Next century challenges: Scalable coordination in sensor networks". In IEEE/ACM MobiCom'99, pages 263.270, August 1999.

[3] M. Alanyali, V. Saligrama, O. Savas, and S. Aeron, "Distributed Bayesian hypothesis testing in sensor networks", Proceedings of the 2004 American Control Conference, 6:5369-5374, 2004.

[4] R. Olfati-Saber, E. Franco, E. Frazzoli, and J. S. Shamma, "Belief consensus and distributed hypothesis testing in sensor networks", Workshop on Network Embedded Sensing and Control, Notre Dame University, Oct. 2005.

[5] R. Olfati-Saber and R. M. Murray, "Consensus problems in networks of agents with switching topology and time-delays", IEEE Transactions on Automatic Control, Volume 49, Issue 9, Sept. 2004

[6] S. Boyd, A. Ghosh, B. Prabhakar, D. Shah, "Randomized Gossip Algorithms," IEEE/ACM Transactions on networking, vol. 14, issue SI, pp. 2508-2530, June 2006.

[7] Dimakis, A. D. G.; Sarwate, A. D.; Wainwright, M. J, "Geographic Gossip: Efficient Averaging for Sensor Networks", IEEE Transactions on Signal processing, March 2008 .

[8] Aysal, Tuncer C.; Yildiz, Mehmet E.; Sarwate, Anand D.; Scaglione, Anna, "Broadcast gossip algorithms: Design and analysis for consensus", Decision and Control, 2008.

[9] J. Perl. Probabilistic reasoning in intelligent systems: networks of plausible inference. Morgan Kaufmann, 1988.

[10] L. Xiao, S. Boyd, and S. Lall, "Distributed average consensus with timevarying metropolis weights," submitted to Automatica, June 2006.

[11] Saligrama, V. Alanyali, M. Savas, O.Savas, "Distributed Detection in Sensor Networks With Packet Losses and Finite Capacity Links", IEEE Transaction on Signal Processing, vol. 54, pp. 4118-4132, 2006. 